\title{
Evaluation of the effects of L-Theanine on neurobehavior in an adult male Sprague-Dawley Rat Model of PTSD
}

\author{
John E Buonora, Patrick M Krum \& Tomás Eduardo Ceremuga* \\ US Army Graduate Program in Anesthesia Nursing, Academy of Health Sciences, AMEDDC\&S, 3490 Forage Rd., Ste 112, Fort Sam \\ Houston, Texas 78234-7585, United States of America
}

\section{Article history}

Received: 25 April 2019

Accepted: 15 June 2019

Published: 16 July 2019

\section{Publisher}

Horizon e-Publishing Group

\section{*Correspondence}

Tomás Eduardo Ceremuga

Thomas.E.Ceremuga.civ@mail.mil

\begin{abstract}
Post-traumatic stress disorder (PTSD) continues to be one of the most common mental health disorders in the United States and may occur in response to traumatic experiences. Currently, there are no interventions that prevent the development of PTSD. L-Theanine (L-Th), a major compound in green tea has been found to decrease anxiety and prevent memory impairment and may have potential effects in the prevention of PTSD. Sixty rats were divided into six experimental groups: control vehicle, control L-Th, control naïve, PTSD vehicle, PTSD Pre-L-Th (prophylactic), PTSD Post-L-Th (non-prophylactic). PTSD was induced by a 3-day restraint/tail shock stress model. The effects of L-Th on neurobehavior were evaluated by Elevated Plus-Maze (EPM), Morris Water Maze (MWM), and Forced Swim Test (FST). Our study found that the total food intake weight of PTSD Pre-L-Th (prophylactic) rats were significantly increased compared to that of PTSD vehicle rats $(p=.04)$. Administration of L-Th 24 hours before the initial PTSD event or for 10 days following the last PTSD stress event did not statistically improve mean open arm exploration on the EPM, spatial memory, and learning in the MWM or behavioral despair measured by the FST $(p>0.05)$. Although the 3-day restraint/tail shock stress model caused stress in the rodents, it did not produce reported PTSD-like anxiety and depression or spatial memory loss. The effect of Pre-L-Th or Post-L-Th treatment, on the neurobehavioral functions could not be effectively evaluated. However, this study provides a foundation for future studies to try different rodent PTSD models to induce PTSD-like neurobehavioral impairments to explore dosage, frequency, as well as the duration of L-Th administration before and/or after the post-traumatic event. The 3-day restraint/tail shock stress model caused stress in the rodents, Pre-L-Theanine treatment preconditioned the PTSD rats to endure stress.
\end{abstract}

Keywords: PTSD; Neurobehavior; L-Theanine; Green Tea; Rat.

Citation: Buonora JE, Krum PM, Ceremuga TE. Evaluation of the effects of L-Theanine on neurobehavior in an adult male Sprague-Dawley Rat Model of PTSD Plant Science Today 2019; 6(3):299-308 https://doi.org/10.14719/pst.2019.6.3.564

Copyright: (C) Buonora et al (2019). This is an open-access article distributed under the terms of the Creative Commons Attribution License, which permits unrestricted use, distribution, and reproduction in any medium, provided the original author and source are credited (https://creativecommons.org/licenses/by/4.0/).

Indexing: Plant Science Today is covered by Scopus,Web of Science, BIOSIS Previews, ESCI, CAS, AGRIS, CABI, Google Scholar, etc. Full list at http://www.plantsciencetoday.online

\section{Introduction}

Post traumatic stress disorder (PTSD) may happen after exposure to traumatic events, which is one of the most prevalent mental health disorders in the United States (1). PTSD can run a chronic course with many debilitating symptoms. While the reported lifetime prevalence of PTSD in the US is about $8.3 \%$ (2), higher prevalence rates of PTSD are reported in veterans who served in different combat events. PTSD prevalence in Iraq and 
Afghanistan veterans was estimated as high as $20 \%$ to $30 \%$ (3). Longitudinal studies found that many trauma exposed subjects experienced delayed-onset PTSD, which could evolve into a chronic mental health condition (4). A recent study found that 40 years after the Vietnam War, veterans were still experiencing current full PTSD symptoms (5). PTSD is also quite prevalent in civilian populations. In a study with 5,692 respondents in the US, $82.7 \%$ indicated they had experienced exposure to severe and potentially traumatic events, and $8.3 \%$ of those exposed to trauma were diagnosed with lifetime PTSD. Furthermore, across 24-nations study found that as high as $65 \%$ of surveyed subjects reported exposure to traumatic events, and $12 \%$ of these subjects eventually developed PTSD $(6,7)$.

Many symptoms are used for diagnosis of PTSD include recurrent distressing and intrusive memories and disturbing nightmares of the traumatic event, hyper arousal, sleep disturbance, inability to concentrate, emotional dissociation, and avoidance of anything associated with the traumatic occurrence (8). Negative changes in cognition and mood are also part of the diagnostic criteria, including negative beliefs about oneself or the world, negative emotions, and/or impaired memory (9). Many studies found that PTSD is associated with neurocognitive dysfunctions such as verbal learning, working memory, attention, and processing speed (10-12). Many studies have indicated the strong correlation of PTSD with dysfunction in the fronto-limbic networks (i.e., the amygdala, hippocampus, and cingulate cortex) (1115).

The amygdala and hippocampus play important roles in processing emotional memory and the amygdala is especially linked to negative emotional responses (16). Decreased volume in the hippocampus, anterior cingulate cortex, and amygdala have been identified in many PTSD studies $(15,17-20)$.

The evolvement of PTSD tends to be gradual and lasts for a long period of time. Many behavioral and cognitive disabilities severely compromise the quality of work, family, and social life; and these pathological changes and debilitating comorbidities may also turn into irreversible outcomes $(21,22)$. There is an urgent need to explore new pharmacological and nutraceutical interventions for PTSD. Due to the difficulty of obtaining clinical samples, animal models have been widely used to do PTSD research. Animal models can be developed to mimic core features of human PTSD, such as susceptibility to various stressors (23), avoidance (24), hyperarousal (25), fear dysregulation (generalization and deficient extinction) $(25,26)$ and hippocampal dysfunction $(27,28)$. Thus, wellvalidated rodent experimental models are a great tool to translate the rodent data to the human physiology and neurochemical changes associated with anxiety, depression, spatial learning, and memory formation.

Although progress has been made in understanding and treating symptoms of PTSD during the past few decades, more effective pharmacological and nutraceutical compounds are still needed because of the pathophysiological complexity of PTSD. One such medication compound is L-Theanine (L-Th). Sakato first discovered and isolated the glutamic acid analog and non-protein forming amino acid, L-Th, from green tea in 1949 (29). L-Th is also an analog to the excitatory neurotransmitter glutamate and glutamine. Because of its ability to cross the blood brain barrier (30), L-Th had been shown to decrease anxiety by enhancing the production of the inhibitory, relaxing neurotransmitter GABA, while blocking the excitatory glutamate receptors in the brain (31). Our early study showed that L-Th affects the expression of genes in brain areas responsible for fear and aggression (amygdala) and memory (hippocampus), helping to balance the behavioral responses to PTSD (32). L-Th is also neuroprotective by preventing memory impairment $(33,34)$. A rodent model study showed pre-stress treatment with L-Th not only reversed cognitive impairment and oxidative damage, but also reversed the abnormal levels of stress hormones (35). We were interested in evaluating the effects of L-Th as a prophylactic treatment and as a post-traumatic stress event treatment in a well-established rodent model.

\section{Material and Methods}

\section{Animals}

The study used 200-249 grams adult male SpragueDawley rats (Envigo, Houston, TX). Handling of the animals was only for weighing, drug administration, and cleaning of cages. After the acclimation period, body weight, food, and water consumption were measured daily up to the first day of neurobehavioral testing. Rats were randomly assigned to the non-stressed group or the 3-day restraint shock PTSD group. The three non-stressed subgroups consisted of: control vehicle, control L-Th, and control naïve. The three stressed groups consisted of PTSD-vehicle, PTSD Pre-L-Th, and PTSD Post- L-Th. Each animal received a subcutaneous injection of either saline (vehicle) or L-Th $10 \mathrm{mg} / \mathrm{kg}$ in saline (L-Th Catalog \# 459340050 was purchased from Acros Organics, New Jersey) twice per day (0700 hrs and $1900 \mathrm{hrs}$ ). The Pre-L-Th animals were given their first dose 24 hours before the 3-day restraint/shock stress model began, while the Post-L-Th animals were given their first dose of L-Th following the third day of the restraint/shock. All animal experiments were performed in accordance with the Association for Assessment and Accreditation of Laboratory Animal Care (AAALAC International) directives after obtaining the approval from the US 
Army Institute of Surgical Research Institutional Animal Care and Use Committee (IACUC). The IACUC at the US Army Institute of Surgical Research, Fort Sam Houston approved of this research protocol. Protocol \#A-15-037.

\section{Three-day Restraint/tail Shock PTSD Stress Model}

Stress exposure consisted of one two-hour session per day of immobilization and tail-shocks, as previously described in our work (36). Rats were restrained in a transparent acrylic glass tube and 40 electric shocks ( $2 \mathrm{~mA}$, 3s duration) were applied at random intervals (140 - 180s). Commercial hardware and software controlled the timing and amplitude of the stimulus (Precision Animal Shocker, Coulbourn Instruments, Columbus, Ohio, USA). Rats were stressed for three consecutive days at approximately the same time each day. It has been demonstrated that repeated stress sessions are more effective than a single stress session in producing physiological and behavioral abnormalities such as acoustic startle response and reduced body weight consistent with PTSD (37). After the 3-day restraint/tail shock stress model was completed, all rats except the control naïve rats were given subcutaneous injections based on their body weight twice daily for a period of ten days. This allowed for the development of PTSD-like symptoms, as demonstrated by previous studies (37).

\section{Elevated Plus Maze (EPM)}

The EPM is a widely utilized and validated instrument to measure anxiety in the rodent model (38). As cited in our previous manuscript (36), the EPM is made entirely of Plexiglas ${ }^{\circledR}$ and consists of a set of opposing open arms and a set of opposing closed arms $(50 \mathrm{~cm} \times 10 \mathrm{~cm})$. The two open arms are lined with $1 \mathrm{~cm}$ high Plexiglas ${ }^{\circledR}$ on the sides to avoid falls. The maze is placed 50 centimeters above the floor and surrounded by screens to minimize any room cues or influences. The walking surface of the EPM is Plexiglas $^{\circledR}$ to avoid excess stimulation and provide a waterproof surface when exposed to urine and feces. The maze is in the shape of a cross (+), with the intersection of the four arms measuring 10 x $10 \mathrm{~cm}$. Each experimental session was recorded for five minutes by the AnyMaze $^{\circledR}$ software where mean speed (centimeters per second), mean time mobile (seconds), and open-arm time ratio (\%) were the main data points investigated. Open arm time ratio was calculated by taking the time spent in the open arm divided by the total recorded time and multiplying by 100 to get a percentage. An increase in the percentage of time spent in the open arms reflects an anxiolytic effect (38). The EPM was cleansed between each testing with non-fragrant soap and water and thoroughly dried. After the EPM test, animals were taken to the Morris water maze room.

\section{Morris Water Maze (MWM)}

After completion of the five-minute EPM, the animals were taken to the MWM for testing. The water maze task has been most extensively used to investigate specific aspects of spatial memory. This task is based upon the premise that animals have evolved an optimal strategy to explore their environment and escape from the water with a minimum amount of effort - i.e., swimming the shortest distance possible (39). The time it takes a rat to find a hidden platform in a water pool after previous exposure to the setup, using only available external cues, is determined as a measure of spatial memory (40). The water maze test, as described previously with minor modifications, was used on all animals (41, 42). The setup consisted of a circular tank $(190 \mathrm{~cm}$ in diameter) filled with water (up to $30 \mathrm{~cm}$ deep; temperature: $22 \pm 2{ }^{\circ} \mathrm{C}$ ) and made opaque by the addition of a non-toxic dye. The pool was divided into four zones. A platform $(18 \mathrm{~cm} \times 18 \mathrm{~cm})$ was submerged $2 \mathrm{~cm}$ below the water surface in zone 1. The pool was placed in a small room with external cues, kept constant throughout the experiments. The data were recorded and analyzed with an overhead video-camera connected to the AnyMaze ${ }^{\circledR}$ software. For training, all rats were exposed six times per day to the setup for two consecutive days, for a total of 12 trials. Each rat was given 60 seconds to randomly explore the water maze. If the animal did not find the platform in this period by chance, it was guided to it and allowed to remain there for 10 seconds to familiarize itself with the location of the platform relative to the visual cues in the maze. Formal data recording consisted of the probe test and commenced on the third day of MWM exposure. The probe test involved removing the platform and the rats undergoing a single trial of 60 seconds (probe trial). The percentage of time spent in each zone and the area where the platform was previously located was recorded. After the end of the probe test, animals were taken to the forced swim test room.

\section{Forced Swim Test (FST)}

After testing in the MWM, animals were carried to a separate room and evaluated in the FST. The FST is a validated test of behavioral despair in the rodent model (43). In two sessions separated by 24 hours, rats were forced to swim in a narrow cylinder from which they cannot escape. Transparent glass cylinders $(20.32 \mathrm{~cm} \times 71.12 \mathrm{~cm})$ contained water $\left(25 \pm 2^{\circ} \mathrm{C}\right)$ at a depth sufficient to prevent the rat from touching the bottom of the cylinder. A 15 minute training session was conducted 24 hours prior to data collection and without behavioral recording. The initial 15minute session was conducted to habituate and acclimate the rats to the test situation, thereby providing a stable, high level of immobile behavior during the five-minute recorded test session 24 hours later. The AnyMaze ${ }^{\circledR}$ software was used to 
video record the five-minute test session. The deidentified videos were independently reviewed by three investigators and the amount of time the rats were immobile was the mean of the three investigators' times. The measurement of the duration of immobility when rodents are exposed to an inescapable situation (FST) is a reflection of behavioral despair or learned helplessness. In this rodent model of depression, the longer the duration of immobility, the greater the behavioral despair or depression. The amount of immobility time was statistically analyzed between groups.

\section{Statistical analysis}

Each group consisted of 10 subjects $(\mathrm{n}=10)$. For this design, a one-way ANOVA was conducted for each
( $M=313.3$ grams $)$ was significantly lower $(p<.05)$ than that of the control-naïve group ( $M=340.6$ grams), but not different from the control-vehicle group mean weight ( $M=327.6$ grams). When examining the pairwise differences via the LSD hoc test, the mean total body weight of the PTSDPre-L-Th group or the PTSD-Post-L-Th treatment group was not different from that of the PTSDvehicle group (Table 1).

\section{Total water intake}

For the total water intake weight outcome (summed total score), a significant difference between the six groups was found: $F(5,54)=3.7, p$ $=.006\left(\eta^{2}=.255\right)$. The control-naïve group had the highest mean $(M=657.2, S E M=26.5)$ and the

Table 1. Total body weight pre and post restraint shock

\begin{tabular}{|c|c|c|c|c|c|}
\hline Group & Pre-Shock $(M)$ & Post-Shock $(M)$ & Mean Difference & Cohens d & $\eta^{2}$ \\
\hline Control-vehicle & 298.5 & 327.6 & 29.1 & 4.67 & 0.66 \\
\hline Control-L-Theanine & 297.6 & 328.6 & 31.0 & 4.17 & 0.68 \\
\hline Control-naïve & 302.2 & 340.6 & 38.4 & 5.32 & 0.77 \\
\hline PTSD-vehicle ${ }^{*}$ & 302.8 & 313.3 & 10.5 & 2.01 & 0.20 \\
\hline PTSD-Pre-L-Theanine & 297.5 & 310.9 & 13.4 & 1.02 & 0.29 \\
\hline PTSD-Post-L-Theanine & 296.3 & 301.3 & 05.0 & 0.41 & 0.05 \\
\hline
\end{tabular}

$M=$ Mean; ${ }^{*} p<.05$

of the outcome variables, where the means $(M)$ and standard error of the means (SEM) are reported. All assumptions were examined including homogeneity of error variances (via the Levine test) and normality. The eta-squared $\left(n^{2}\right)$ effect size is reported. Though interpreting and casting judgment as to what constitutes a small/medium/large effect size is contextdependent (Pek \& Flora, 2018) (44) using Cohen's (1988) taxonomy .01/.059/138 will be small/medium/large (45). All outliers and data anomalies were examined and addressed accordingly (e.g., transformations, nonparametric options, etc.). In the event of a significant result (a $=.05)$, post-hoc tests (e.g., LSD) were performed.

\section{Results}

\section{Total body weight}

Measurements of the rodents' total body weight before the PTSD shock treatment and before sacrifice were taken and compared between groups. For the $6 \times 2$ (group $\mathrm{x}$ time) design $(n=60)$, a significant 2-way interaction was found: $F(5,54)$ $=21.67, p<.001\left(\eta^{2}=.667\right)$. The pre-shock stage the PTSD-vehicle group had the highest mean ( $M=$ 302.8 grams) and the PTSD-post-treatment group had the lowest ( $M=296.3$ grams), whereas for the subsequent temporal outcome (10 days after restraint/shock) the control-naïve group had the highest mean ( $M=340.6$ grams) and the PTSD-posttreatment group (again) had the lowest ( $M=301.3$ grams) (Table 1).

When compared to the mean total body weight of post PTSD shock, the PTSD-vehicle group
PTSD-post-treatment group the lowest ( $M=517.6$, $S E M=23.7)$. The PTSD-vehicle group water intake weight $(M=537$ grams, $S E M=31.5)$ was significantly lower than that of the control-naïve group $(M=657.2$ grams, $S E M=26.5)(p=.002)$, but not different from that of the control-vehicle group $(M=605.6$ grams, $S E M=10.5)$. When examining the pairwise differences via the LSD hoc test, the total water intake weight of the PTSD-Pre-L-Th group or the PTSD-Post-L-Th treatment group was not different from that of the PTSD-vehicle group (Table 2).

Table 2. Total water intake

\begin{tabular}{lr}
\hline Group & Mean Gram Weight \pm SEM \\
\hline Control-vehicle & $605.6 \pm 10.5$ \\
\hline Control-L-Theanine & $587.8 \pm 7.30$ \\
\hline Control-naïve & $657.2 \pm 26.5$ \\
\hline PTSD-vehicle* & $537.0 \pm 31.5$ \\
\hline PTSD-Pre-L-Theanine & $594.6 \pm 40.8$ \\
\hline PTSD-Post-L-Theanine & $517.6 \pm 23.7$ \\
\hline
\end{tabular}

\section{Total food intake}

For total food intake, a significant difference between the six groups was found: $F(5,54)=2.78$, $p=.026\left(\eta^{2}=.205\right)$. The PTSD-vehicle group had the lowest food intake weight $(M=323.8$ grams, $S E M=$ 3.5). It was significantly lower than that of the control-naïve group ( $p=.002$ ), but not different from that of the control-vehicle group ( $M=381.2$ grams, $S E M=9.5$ ). When examining the pairwise differences via the LSD post hoc, the PTSD-Pre-LTh group food intake weight $(M=360.0$ grams, $S E M=18.8)$ was significantly higher than that of the PTSD-vehicle group ( $p=.04$ ) (Table 3). 


\section{Elevated plus maze}

There were no significant differences between the groups pertaining to mean: distance, mobile episodes, time mobile, mean speed, maximum speed, or open arm entries. Analysis of the ratio for open arm time versus total time spent in the elevated plus maze: although the mean ratio of PTSD-vehicle group was significantly lower than that of the control-naïve group ( $p=.046$ ), but not different from that of the control vehicle group. No difference was found in the ratio of open arm time versus total time spent in the elevated plus maze when comparing the PTSD-pre-treatment group or the PTSD-post-treatment group to the PTSD-vehicle group (Fig 1).

Table 3. Total food intake

\begin{tabular}{lr}
\hline Group & Mean Gram Weight \pm SEM \\
\hline Control-vehicle & $352.2 \pm 05.7$ \\
\hline Control-L-Theanine & $347.0 \pm 11.5$ \\
\hline Control-naïve & $381.2 \pm 9.5$ \\
\hline PTSD-vehicle $^{* \#}$ & $323.8 \pm 3.5$ \\
\hline PTSD-Pre-L-Theanine & \\
\hline PTSD-Post-L-Theanine & $360.0 \pm 18.8$ \\
\hline
\end{tabular}

SEM $=$ Standard Error of the Mean; ${ }^{*} p=.002 ;{ }^{*} p=.04$

\section{Morris water maze}

When analyzed the test result from the Morris water maze, no statistically significant differences were found when comparing distance traveled, mean speed, max speed, zone 1 distance traveled, zone 1 latency to first entry, zone 1 max speed, and entries to the platform area in zone 1 (the platform zone) between groups. Similarly, the Time in Zone 1 (Platform Zone) Time outcome, no significant difference was found between the six groups: $F(5$, 54) $=.78, p=.567\left(\eta^{2}=.067\right)($ Fig 2$)$.

\section{Forced swim test}

For the forced swim test, one subject was eliminated from the L-Th control group $(n=9)$ because of weakness and the inability to complete the FST 15 minute trial. There was not a significant difference between the six groups: $F(5,53)=1.27, p$ $=.291\left(\eta^{2}=.107\right)$ in term of the Mean Time Mobile outcome. When examining the pairwise differences via the LSD hoc test, the PTSD-pre-treatment group had a significantly higher mean $(M=48.17, S E M=$ 9.26) than that of the control- naïve group ( $M=$ 28.53, $S E M=6.69)(p=0.044)($ Fig 3$)$.

\section{Discussion}

PTSD continues to be a devastating psychiatric disorder of high prevalence. The goal of this study was to evaluate if L-Th, given to stressed rats in a rodent model of PTSD, before and after the induction of PTSD, has neurobehavioral effects. The prophylactic and non-prophylactic effects of LTh, on PTSD-like symptoms such as anxiety, behavioral despair, and alterations in learning and spatial memory were evaluated.

Due to the difficulty of obtaining PTSD patient samples, animal models have been widely used to compensate for the need for PTSD human disease research. Many different animal models have been developed to delineate the underlying neurobiological abnormalities of human PTSD. A physical stressor inducing PTSD is one of the common rodent PTSD models. Our study used both restraint and shock to induce PTSD, studies have showed the stress caused by this modeling can closely parallel the depression, anxiety, and fear in PTSD patients $(46,47)$. These physical stressors can induce neuro-structural and function changes in the hippocampus and/or amygdala which are responsible for these PTSD abnormalities (48). Our study found that rats exposed to PTSD stress lost weight, compared to non-stressed rodents, the PTSD group also had lower water and food intake compared to that of the non-stressed rodents, indicating that the rats in the PTSD groups were under chronic stress as shown in our previous work (36). Administration of L-Th prior to PTSD events prevented the decrease in food consumption, significantly increased food intake compared to that of the PTSD-vehicle group. This suggests that administration of L-Th prior to rodent exposure to PTSD shock preconditioned the rodents to endure stressful events.

The EPM has been a standard measurement of rodent anxiety by measuring the time of the rodent's exploration of novel and unprotected environments. It has been shown that L-Th can cross the blood-brain barrier easily and quickly and L-Th reached its peak concentrations in mammals after 30 to 120 min after ingestion (49, 50). Similarly, L-Th can be absorbed very quickly in various resource formats, and reach maximum concentration in humans within 50 minutes after ingestion (51). Several human studies found that LTh was beneficial for reducing anxiety and improving negative moods caused by high blood pressure or cancer (52-54). So it is hypothesized that L-Th may be protective to reduce PTSD related stress. Although the PTSD group showed anxietylike behavior by having a decrease in mean open arm time compared to non-stressed rodents, our model did not produce severe anxiety stress as reported after rodents were exposed to 1-hour restraint stress in a 14 day consecutive stress model (55); or by exposing rats to under water stress to induce an occurrence rate of $53 \%$ of extreme anxiety (56). We did not see any protective effect from pre or post-treatment of LTh because of the lack of severe anxiety symptom in our rodents. A prolonged repeat stress model or a different stress model may be needed to evaluate L-Th effects.

In a rat model of repeated cerebral ischemia, theanine was administered intraperitoneally after the first ischemic insult 


\section{Ratio Open Arm Time to Total Time on Elevated Plus Maze}

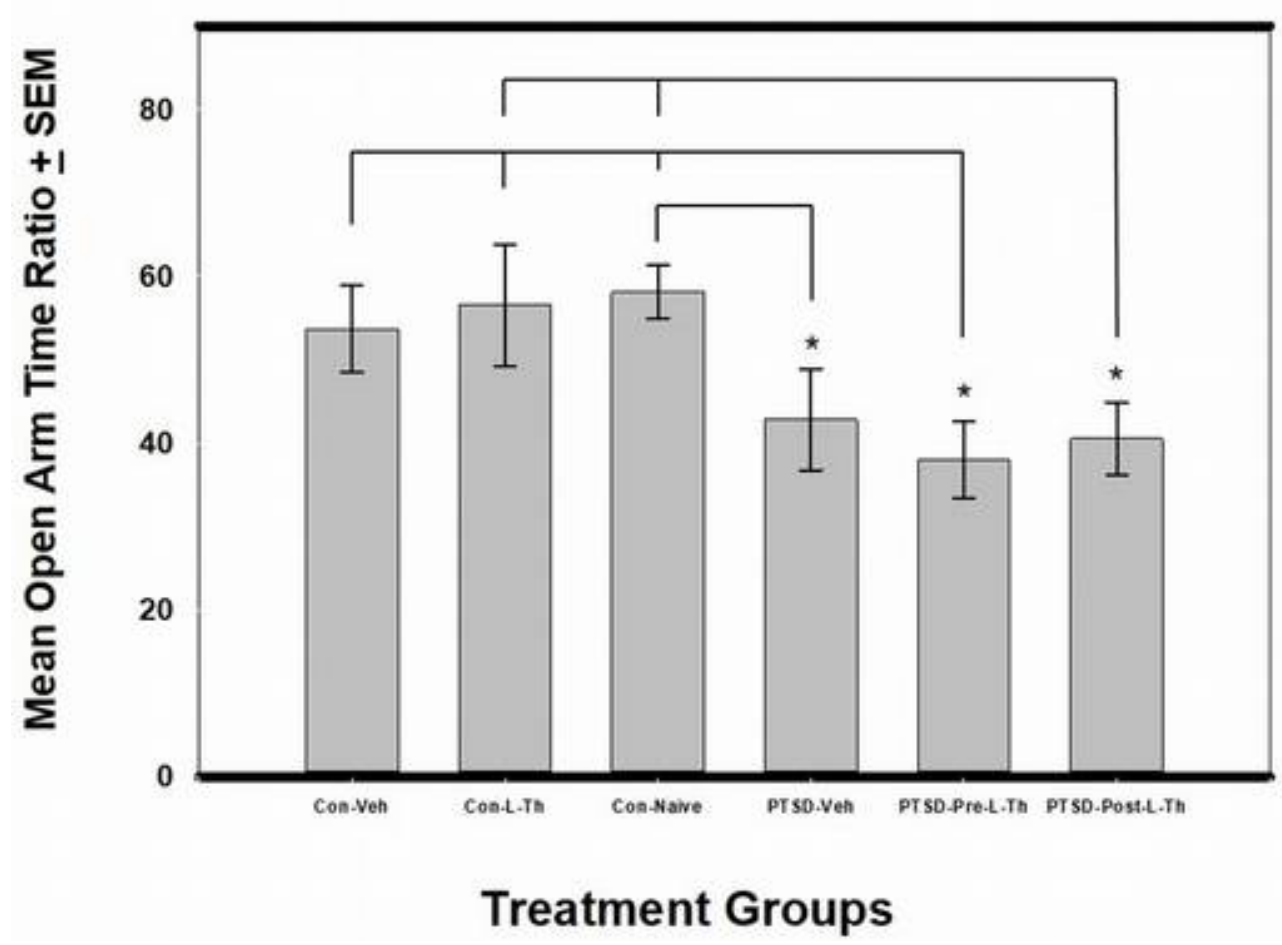

Fig. 1. ANOVA of mean open-arm time ratio from elevated plus-maze (EPM) and standard error of the mean (SEM). Each of the main two groups (Stressed and Non-stressed) had three subgroups: non-stressed: control vehicle, control L-Th, control naive, and stressed: Posttraumatic stress disorder (PTSD) vehicle, PTSD Pre-L-Th (prophylactic), PTSD Post-L-Th (non-prophylactic). Mean Open-Arm Time Ratio is reported with the SEM. Asterisks denote statistical significance at $\mathrm{p}<.05$.

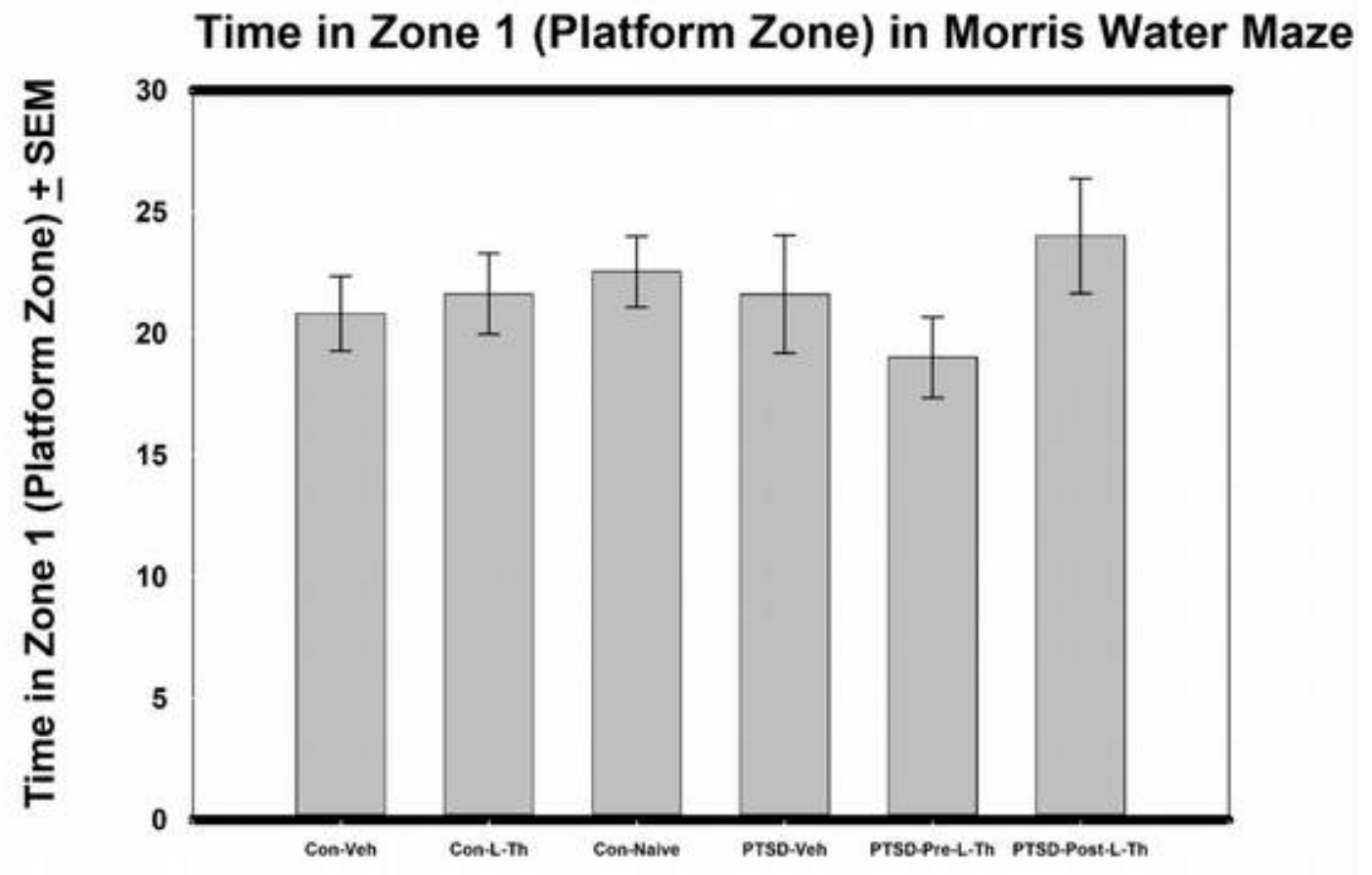

\section{Treatment Groups}

Fig. 2. ANOVA of time in zone1 (Platform Zone) from Morris water maze (MWM) and standard error of the mean (SEM). Each of the main two groups (Stressed and Non-stressed) had three subgroups: non-stressed: control vehicle, control L-Th, control naive, and stressed: Posttraumatic stress disorder (PTSD) vehicle, PTSD Pre-L-Th (prophylactic), PTSD Post-L-Th (non-prophylactic). Mean time (seconds) in Zone 1 (Platform Zone) is reported with the SEM. There was not a significant difference between the six groups.

which reversed the impairment of spatial memory caused by the ischemic event (33). A study evaluated the effect of the combination of green tea and L-Th on patients with mild cognitive impairment and found that the green tea/L-Th combination significantly increased memory and 


\section{Mean Time Mobile in the Forced Swim Test}

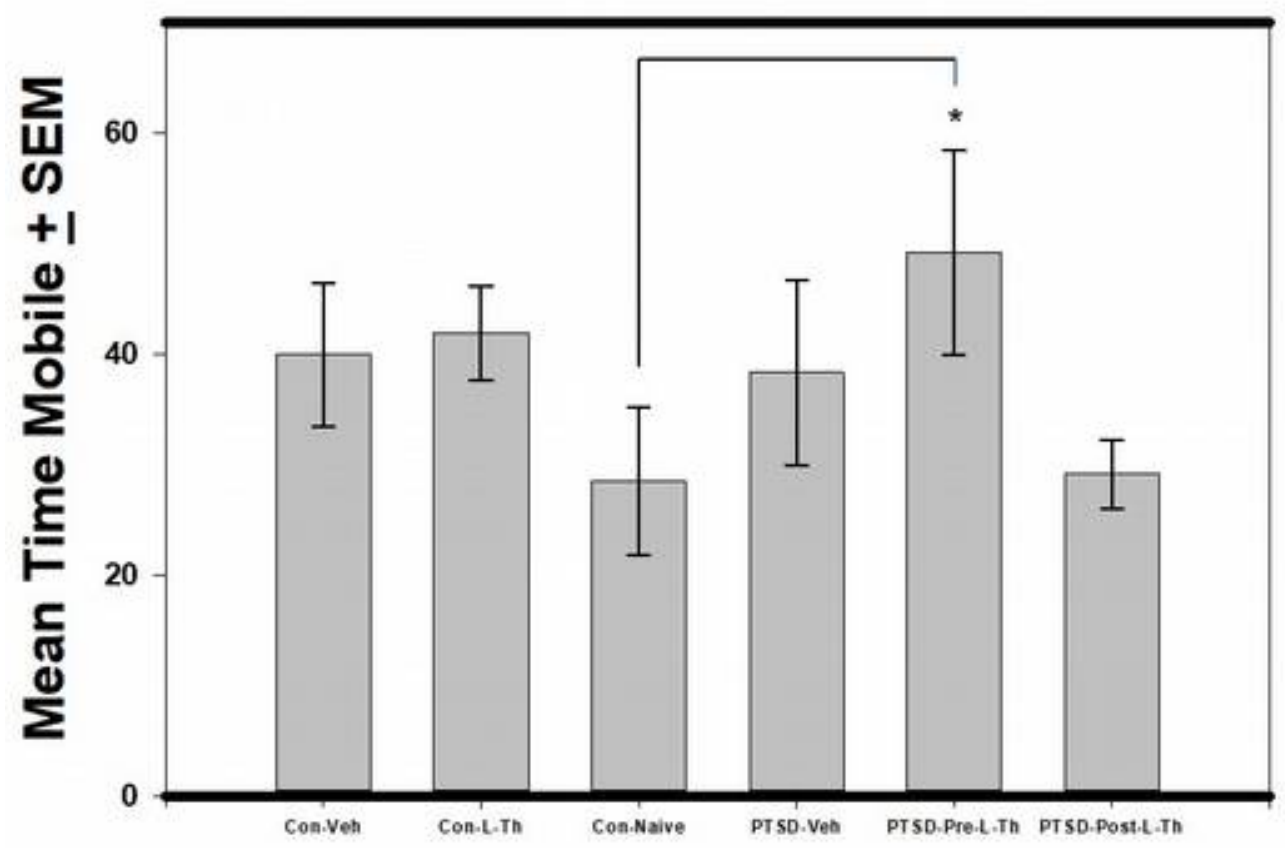

\section{Treatment Groups}

Fig 3. ANOVA of mean time mobile from forced swim test (FST) and standard error of the mean (SEM). Each of the main two groups (Stressed and Non-stressed) had three subgroups: non-stressed: control vehicle, control L-Th, control naive, and stressed: Posttraumatic stress disorder (PTSD) vehicle, PTSD Pre-L-Th (prophylactic), PTSD Post-L-Th (non-prophylactic). Mean time mobile is reported with the standard error of the mean (SEM).Asterisks denote statistical significance at $\mathrm{p}<.05$. PTSD-Pre-L-Th was significantly higher than the control naive.

attention of these patients (57). PTSD is a very complex disorder, many rodent PTSD models showed that trauma induced behavioral and memory deficits can occur both immediately and weeks after the traumatic events $(58,59)$. It has been shown that the MWM measured memory deficit in the rats with a PTSD-like underwater trauma, could have an onset as late as three weeks after the PTSD trauma (46). The 3-day restraint/tail shock stress rat PTSD model used in our study did not significantly impair the memory of rodents in the PTSD group measured by the MWM. Another stress model such as the underwater trauma model $(59,60)$ may be used to produce the PTSDlike memory loss, and different lengths of pre and post treatment can be implemented to assess the prophylactic and non-prophylactic effects of L-Th on spatial memory and learning.

Depression is one of the major symptoms of PTSD. Learned helplessness has been widely used as a rodent model of depression (61). When the rodents were exposed to uncontrollable shock, rodents became inactive and passively tolerant of stress. The learned inactivity or helplessness is parallel to symptoms of depressed patients, so the learned helplessness has been modeled to study the cognitive deficits related to depression (62). In our study, we used the forced swim test to assess depression-like behavioral despair and the effect of L-Th in the prevention and treatment. A rat model of exposure to even a 15 minute session of inescapable foot shocks could result in impaired mobility and attention lasting for weeks (63). In the inescapable foot shock model, animals also showed learning impairment, and this learning impairment was evident even 28 days after the rats' exposure to electric foot shock (64). Our 3-day restraint/tail shock stress model did not cause significant learned helplessness (LH) or depression in rodents of the PTSD group measured by the forced swim test. We may need to use an alternative PTSD model to induce significant LH or depression to evaluate L-Th effects.

Elevated Plus-Maze, Morris Water Maze, and Forced Swim Test were used to assess the effects of L-Th on neurobehaviors in our rat model of PTSD. Our study indicated that Pre-L-Theanine treatment preconditioned the PTSD rats to endure stress, but did not improve their neurobehavioral functions. However, this study provides a foundation for future PTSD treatment explorations. There is no single model of PTSD that can mimic all behavioral symptoms of PTSD. Future studies may be designed to use multiple 
rodent PTSD models to induce different PTSD-like neurobehavioral impairments to explore dosage, frequency, and duration of L-Theanine administration before and/or after the posttraumatic event. Furthermore, biomarkers and gene expression profiling may assist in correlating and identifying potential treatment approaches.

\section{Conclusion}

The 3-day restraint/tail shock stress model caused stress in the rodents, our study found that the total food intake weight of PTSD Pre-L-Th (prophylactic) rats had significantly increased compared to that of PTSD vehicle rats $(p=.04)$. This indicated that Pre-L-Theanine treatment preconditioned the PTSD rats to endure stress. Because the 3-day restraint/tail shock stress model did not produce PTSD-like anxiety, depression or spatial memory loss, the effect of L-Th on the neurobehavioral functions could not be effectively evaluated.

\begin{abstract}
Abbreviations
AAALAC: Association for Assessment and Accreditation of Laboratory Animal Care; ANOVA: Analysis of variance; ${ }^{\circ} \mathbf{C}$ : Celsius; cm: centimeter; EPM: Elevated Plus-Maze; F: F statistic in ANOVA; FST: Forced Swim Test; GABA: Gamma aminobutyric acid; hrs: hours; IACUC: Institutional Animal Care and Use Committee; kg: kilogram; LSD: least significant difference; L-Th: L-Theanine; $\boldsymbol{M}$ : Mean; mA: milliamp; $\mathbf{m g}$ : milligram; MWM: Morris Water Maze; $\mathbf{n}$ : sample size; $\boldsymbol{p}$ : $\mathrm{p}$ value; PTSD: Post-traumatic stress disorder; s: second; SEM: standard error of the mean; US: United States; $\boldsymbol{\eta}^{2}$ : eta-squared.
\end{abstract}

\section{Authors' contributions}

All authors participated in the design, planning, implementation, and data collection of the study. All authors were also instrumental in the writing of the manuscript.

\section{Acknowledgements}

The researchers would like to acknowledge their families and the support they provided, as well as the US Army Graduate Program in Anesthesia Nursing. We would also like to thank Alberto Mares, Roger Chavez, and David Trapolsi for their assistance in the laboratory. Ms. Kathleen McKay, VSB facility manager at US Army Institute of Surgical Research, support staff, and the US Army Institute of Surgical Research for allowing us to conduct this research at their facility. We also thank Dr. Dale Glaser for his expert statistical analysis. This work was funded and supported by a TriService Nursing Research Program Grant.

\section{Competing Interest and Funding Source}

There are no competing interests or conflicts of interest. This work has been funded and supported by the TriService Nursing Research Program.

\section{Disclaimer}

The views expressed in this article are those of the authors and do not reflect the official policy or position of the Department of the Army, Department of Defense, or the US Government.

\section{References}

1. Kessler RC, Petukhova M, Sampson NA, Zaslavsky AM, Wittchen HU. Twelve-month and lifetime prevalence and lifetime morbid risk of anxiety and mood disorders in the United States. Int J Methods Psychiatr Res. 2012;

https://doi.org/10.1002/mpr.1359

21(3):169-84

2. Kilpatrick DG, Resnick HS, Milanak ME, Miller MW, Keyes KM, Friedman MJ. National estimates of exposure to traumatic events and PTSD prevalence using DSM-IV and DSM-5 criteria. J Trauma Stress. 2013; 26(5):537-47. https://doi.org/10.1002/jts.21848

3. Eber S, Barth S, Kang H, Mahan C, Dursa E, Schneiderman A. The National Health Study for a New Generation of United States Veterans: methods for a large-scale study on the health of recent veterans. Mil Med. 2013; 178(9):966-9 https://doi.org/10.7205/MILMED-D-13-00175

4. Sareen J. Posttraumatic stress disorder in adults: impact, comorbidity, risk factors, and treatment. Can J $\begin{array}{lll}\text { Psychiatry. } & \text { 2014; } & \text { 59(9):460-7. }\end{array}$ https://doi.org/10.1177/070674371405900902

5. Marmar CR, Schlenger W, Henn-Haase C, Qian M, Purchia E, Li M, et al. Course of Posttraumatic Stress Disorder 40 Years After the Vietnam War: Findings From the National Vietnam Veterans Longitudinal Study. JAMA Psychiatry 2015; 72(9):875-81. https://doi.org/10.1001/jamapsychiatry.2015.0803

6. Koenen KC, Ratanatharathorn A, Ng L, McLaughlin KA, Bromet EJ, Stein DJ, et al. Posttraumatic stress disorder in the World Mental Health Surveys. Psychol Med. 2017; 47(13):2260-74. https://doi.org/10.1017/S0033291717000708

7. Stein MB, McQuaid JR, Pedrelli P, Lenox R, McCahill ME. Posttraumatic stress disorder in the primary care medical setting. Gen Hosp Psychiatry. 2000; 22(4):2619. https://doi.org/10.1016/S0163-8343(00)00080-3

8. Association AP. Diagnostic and Statistical Manual of Mental Disorders, Fifth Edition. 5 ed. Washington, D.C.2013.

9. Roca V, Freeman TW. Complaints of impaired memory in veterans with PTSD. Am J Psychiatry. 2001; 158(10):1738-9.

https://doi.org/10.1176/appi.ajp.158.10.1738-a

10. Brewin CR, Kleiner JS, Vasterling JJ, Field AP. Memory for emotionally neutral information in posttraumatic stress disorder: A meta-analytic investigation. J Abnorm Psychol. 2007; 116(3):448-63. https://doi.org/10.1037/0021-843X.116.3.448

11. Scott JC, Matt GE, Wrocklage KM, Crnich C, Jordan J, Southwick SM, et al. A quantitative meta-analysis of neurocognitive functioning in posttraumatic stress 
disorder. Psychol Bull. 2015; 141(1):105-40. https://doi.org/10.1037/a0038039

12. Brailey K, Vasterling JJ. Neuropsychological findings in adults with PTSD. In: Vasterling JJ, Brailey K, editors. Neuropsychology of PTSD: Biological, cognitive, and clinical perspectives. Vasterling JJ, Brailey $\mathrm{K}$, editors. New York: Guilford Press; 2005.

13. Bremner JD, Randall P, Scott TM, Bronen RA, Seibyl JP, Southwick SM, et al. MRI-based measurement of hippocampal volume in patients with combat-related posttraumatic stress disorder. Am J Psychiatry. 1995; 152(7):973-81. https://doi.org/10.1176/ajp.152.7.973

14. Liberzon I, Sripada CS. The functional neuroanatomy of PTSD: a critical review. Prog Brain Res. 2008; 167:151-69. $\underline{6123(07) 67011-3}$

https://doi.org/10.1016/S0079-

15. Morey RA, Gold AL, LaBar KS, Beall SK, Brown VM, Haswell CC, et al. Amygdala volume changes in posttraumatic stress disorder in a large case-controlled veterans group. Arch Gen Psychiatry. 2012; 69(11):1169-78.

https://doi.org/10.1001/archgenpsychiatry.2012.50

16. Etkin A, Wager TD. Functional neuroimaging of anxiety: a meta-analysis of emotional processing in PTSD, social anxiety disorder, and specific phobia. Am J Psychiatry. 2007; 164(10):1476-88. https://doi.org/10.1176/appi.ajp.2007.07030504

17. Hedges DW, Woon FL. Premorbid brain volume estimates and reduced total brain volume in adults exposed to trauma with or without posttraumatic stress disorder: a meta-analysis. Cogn Behav Neurol. 2010; 23(2):124-9 https://doi.org/10.1097/WNN.0b013e3181e1cbe1

18. Karl A, Schaefer M, Malta LS, Dorfel D, Rohleder N, Werner A. A meta-analysis of structural brain abnormalities in PTSD. Neurosci Biobehav Rev. 2006; 30(7):1004-31.

https://doi.org/10.1016/j.neubiorev.2006.03.004

19. Kitayama N, Vaccarino V, Kutner M, Weiss P, Bremner JD. Magnetic resonance imaging (MRI) measurement of hippocampal volume in posttraumatic stress disorder: a meta-analysis. J Affect Disord. 2005; 88(1):79-86. https://doi.org/10.1016/j.jad.2005.05.014

20. Kuhn S, Gallinat J. Gray matter correlates of posttraumatic stress disorder: a quantitative metaanalysis. Biol Psychiatry. 2013; 73(1):70-4. https://doi.org/10.1016/j.biopsych.2012.06.029

21. Scott JC, Harb G, Brownlow JA, Greene J, Gur RC, Ross RJ. Verbal memory functioning moderates psychotherapy treatment response for PTSD-Related nightmares. Behav Res Ther. 2017; 91:24-32. https://doi.org/10.1016/j.brat.2017.01.004

22. Solomon Z, Shklar R, Mikulincer M. Frontline treatment of combat stress reaction: a 20-year longitudinal evaluation study. Am J Psychiatry. 2005; 162(12):2309-14.

https://doi.org/10.1176/appi.ajp.162.12.2309

23. Rudy JW, Huff NC, Matus-Amat P. Understanding contextual fear conditioning: insights from a twoprocess model. Neurosci Biobehav Rev. 2004; 28(7):675-85.

https://doi.org/10.1016/j.neubiorev.2004.09.004

24. Dielenberg RA, McGregor IS. Defensive behavior in rats towards predatory odors: a review. Neurosci

\section{Biobehav Rev. 2001; 25(7-8):597-609. https://doi.org/10.1016/S0149-7634(01)00044-6}

25. Manion ST, Gamble EH, Li H. Prazosin administered prior to inescapable stressor blocks subsequent exaggeration of acoustic startle response in rats. Pharmacol Biochem Behav. 2007; 86(3):559-65. https://doi.org/10.1016/j.pbb.2007.01.019

26. Takahashi T, Morinobu S, Iwamoto Y, Yamawaki S. Effect of paroxetine on enhanced contextual fear induced by single prolonged stress in rats. Psychopharmacology (Berl) 2006; 189(2):165-73. https:// doi.org/10.1007/s00213-006-0545-6

27. Goswami S, Samuel S, Sierra OR, Cascardi M, Pare D. A rat model of post-traumatic stress disorder reproduces the hippocampal deficits seen in the human syndrome. Front Behav Neurosci. 2012; 6:26. https://doi.org/10.3389/fnbeh.2012.00026

28. Woodson JC, Macintosh D, Fleshner M, Diamond DM. Emotion-induced amnesia in rats: working memoryspecific impairment, corticosterone-memory correlation, and fear versus arousal effects on memory. Learn Mem. 2003; 10(5):326-36. https://doi.org/10.1101/lm.62903

29. Sakato Y. The chemical constituents of tea: III. A new amide theanine. Nippon Nogeikagaku Kaishi. 1949; 23:262-7.

30. Gomez-Ramirez M, Higgins BA, Rycroft JA, Owen GN, Mahoney J, Shpaner M, et al. The deployment of intersensory selective attention: a high-density electrical mapping study of the effects of theanine. Clin Neuropharmacol. 2007; 30(1):25-38. https://doi.org/10.1097/01.WNF.0000240940.13876.17

31. Nathan PJ, Lu K, Gray M, Oliver C. The neuropharmacology of L-theanine(N-ethyl-Lglutamine): a possible neuroprotective and cognitive enhancing agent. J Herb Pharmacother. 2006; 6(2):2130. https://doi.org/10.1080/J157v06n02 02

32. Ceremuga TE, Martinson S, Washington J, Revels R, Wojcicki J, Crawford D, et al. Effects of L-theanine on posttraumatic stress disorder induced changes in rat brain gene expression. Scientific World Journal 2014; 2014:419032. https://doi.org/10.1155/2014/419032

33. Egashira $\mathrm{N}$, Ishigami N, Pu F, Mishima K, Iwasaki K, Orito $\mathrm{K}$, et al. Theanine prevents memory impairment induced by repeated cerebral ischemia in rats. Phytother Res. 2008; 22(1):65-8. https://doi.org/10.1002/ ptr.2261

34. Cho HS, Kim S, Lee SY, Park JA, Kim SJ, Chun HS. Protective effect of the green tea component, Ltheanine on environmental toxins-induced neuronal cell death. Neurotoxicology 2008; 29(4):656-62. https://doi.org/10.1016/j.neuro.2008.03.004

35. Tian X, Sun L, Gou L, Ling X, Feng Y, Wang L, et al. Protective effect of l-theanine on chronic restraint stress-induced cognitive impairments in mice. Brain Res. 2013; 1503:24-32. https://doi.org/10.1016/j.brainres.2013.01.048

36. Valdivieso DA, Baughan TG, 2nd, Canavati UM, Rey AM, Trotter CL, Burrell DR, et al. Effects of pregabalin on neurobehavior in an adult male rat model of PTSD. PLOS One. 2018; 13(12):e0209494. https://doi.org/10.1371/journal.pone.0209494

37. Servatius RJ, Ottenweller JE, Natelson BH. Delayed startle sensitization distinguishes rats exposed to one 
or three stress sessions: further evidence toward an animal model of PTSD. Biol Psychiatry. 1995; 38(8):53946. https://doi.org/10.1016/0006-3223(94)00369-E

38. Pellow S, Chopin P, File SE, Briley M. Validation of open:closed arm entries in an elevated plus-maze as a measure of anxiety in the rat. J Neurosci Methods. 1985; 14(3):149-67.

39. Morris R. Developments of a water-maze procedure for studying spatial learning in the rat. J Neurosci Methods. 1984; 11(1):47-60.

40. Jeong DU, Lee J, Chang WS, Chang JW. Identifying the appropriate time for deep brain stimulation to achieve spatial memory improvement on the Morris water maze. BMC Neurosci. 2017; 18(1):29. https://doi.org/10.1016/0165-0270(85)90031-7

41. D'Hooge R, De Deyn PP. Applications of the Morris water maze in the study of learning and memory. Brain Res Brain Res Rev. 2001; 36(1):60-90. https://doi.org/10.1016/S0165-0173(01)00067-4

42. Wenk GL. Assessment of spatial memory using the radial arm maze and Morris water maze. Curr Protoc Neurosci. 2004; Chapter 8: Unit 8 5A. https://doi.org/10.1002/0471142301.ns0805as26

43. Castagne V, Moser P, Roux S, Porsolt RD. Rodent models of depression: forced swim and tail suspension behavioral despair tests in rats and mice. Curr Protoc Neurosci. 2011; Chapter 8: Unit 8 10A. https://doi.org/10.1002/0471142301.ns0810as55

44. Pek J, Flora DB. Reporting effect sizes in original psychological research: A discussion and tutorial. Psychol Methods. 2018; 23(2):208-25. https://doi.org/10.1037/met0000126

45. Cohen J. Statistical power analysis for the behavioral sciences. 2nd ed. Hillsdale, N.J.: L. Erlbaum Associates: 1988. p.567.

46. Schoner J, Heinz A, Endres M, Gertz K, Kronenberg G. Post-traumatic stress disorder and beyond: an overview of rodent stress models. J Cell Mol Med. 2017; 21(10):2248-56. https://doi.org/10.1111/jcmm.13161

47. Barone FC, Barton ME, White RF, Legos JJ, Kikkawa H, Shimamura M, et al. Inhibition of phosphodiesterase type 4 decreases stress-induced defecation in rats and mice. $\quad$ Pharmacology. 2008; 81(1):11-7. https://doi.org/10.1159/000107662

48. Goswami S, Rodriguez-Sierra O, Cascardi M, Pare D. Animal models of post-traumatic stress disorder: face validity. Front Neurosci. 2013; 7:89. https://doi.org/10.3389/fnins.2013.00089

49. Yokogoshi H, Kobayashi M, Mochizuki M, Terashima T. Effect of theanine, r-glutamylethylamide, on brain monoamines and striatal dopamine release in conscious rats. Neurochem Res. 1998; 23(5):667-73. https://doi.org/10.1023/A:1022490806093

50. Unno T, Suzuki Y, Kakuda T, Hayakawa T, Tsuge H. Metabolism of theanine, gamma-glutamylethylamide, in rats. J Agric Food Chem. 1999; 47(4):1593-6. https://doi.org/10.1021/jf981113t

51. van der Pijl P, Chen L, Mulder TPJ. Human disposition of L-theanine in tea or aqueous solution. Journal of Functional Foods. 2010; 2(4):239-44. https://doi.org/10.1016/j.jff.2010.08.001
52. Kimura K, Ozeki M, Juneja LR, Ohira H. L-Theanine reduces psychological and physiological stress responses. Biol Psychol. 2007; 74(1):39-45. https://doi.org/10.1016/j.biopsycho.2006.06.006

53. Yoto A, Motoki M, Murao S, Yokogoshi H. Effects of Ltheanine or caffeine intake on changes in blood pressure under physical and psychological stresses. J Physiol Anthropol. 2012; 31:28. https://doi.org/10.1186/1880-6805-31-28

54. Shacham S. A shortened version of the Profile of Mood States. J Pers Assess. 1983; 47(3):305-6. https://doi.org/10.1207/s15327752jpa4703 14

55. Shoji H, Mizoguchi K. Acute and repeated stress differentially regulates behavioral, endocrine, neural parameters relevant to emotional and stress response in young and aged rats. Behav Brain Res. 2010; 211(2):169-77. https://doi.org/10.1016/j.bbr.2010.03.025

56. Ritov G, Boltyansky B, Richter-Levin G. A novel approach to PTSD modeling in rats reveals alternating patterns of limbic activity in different types of stress reaction. Mol Psychiatry. 2016; 21(5):630-41. https://doi.org/10.1038/mp.2015.169

57. Park SK, Jung IC, Lee WK, Lee YS, Park HK, Go HJ, et al. A combination of green tea extract and l-theanine improves memory and attention in subjects with mild cognitive impairment: a double-blind placebocontrolled study. J Med Food. 2011; 14(4):334-43. https://doi.org/10.1089/jmf.2009.1374

58. Deslauriers J, Toth M, Der-Avakian A, Risbrough VB. Current Status of Animal Models of Posttraumatic Stress Disorder: Behavioral and Biological Phenotypes, and Future Challenges in Improving Translation. Biol Psychiatry. 2018; 83(10):895-907. https://doi.org/10.1016/j.biopsych.2017.11.019

59. Richter-Levin G. Acute and long-term behavioral correlates of underwater trauma--potential relevance to stress and post-stress syndromes. Psychiatry Res. 1998; 79(1):73-83. $\quad$ https://doi.org/10.1016/S01651781(98)00030-4

60. Wang J, Akirav I, Richter-Levin G. Short-term behavioral and electrophysiological consequences of underwater trauma. Physiol Behav. 2000; 70(3-4):32732. https://doi.org/10.1016/S0031-9384(00)00274-2

61. Seligman ME. Learned helplessness. Annu Rev Med. 1972; 23:407-12. https://doi.org/10.1146/annurev.me.23.020172.002203

62. Vollmayr B, Gass P. Learned helplessness: unique features and translational value of a cognitive depression model. Cell Tissue Res. 2013; 354(1):171-8. https://doi.org/10.1007/s00441-013-1654-2

63. Van Dijken HH, Van der Heyden JA, Mos J, Tilders FJ. Inescapable footshocks induce progressive and longlasting behavioural changes in male rats. Physiol Behav. 1992; 51(4):787-94 https://doi.org/10.1016/0031-9384(92)90117-K

64. Valerio dos Santos C, Gehm T, Hunziker MH. Learned helplessness in the rat: effect of response topography in a within-subject design. Behav Processes. 2011; 86(2):178-83

https://doi.org/10.1016/j.beproc.2010.11.005 